\title{
Patients With Obesity Exhibit a Plateau Pattern of the Right Ventricular Waveform
}

\author{
Yoh Arita $^{\text {a, b }}$, Masako Okada ${ }^{a}$, Nobuyuki Ogasawara ${ }^{a}$, Shinji Hasegawa ${ }^{a}$
}

\begin{abstract}
Background: Ventricular waveforms are characterized by a dip-andplateau pattern during diastole owing to an abrupt termination of ventricular filling because of pericardial constraint under conditions such as constrictive pericarditis (CP). However, constrictive hemodynamics is not specifically caused by CP. Therefore, this study aimed to evaluate whether patients with obesity exhibited constrictive hemodynamics.
\end{abstract}

Methods: Overall, 60 consecutive Japanese patients (mean age, 69.5 years; $45 \%$ women) who underwent right heart catheterization at the Japan Community Healthcare Organization Osaka Hospital from July 2016 to September 2018 were examined. Two-dimensional echocardiography was used to measure the epicardial adipose tissue (EAT) in the standard parasternal long-axis view during end-diastole. Because patients who underwent open-heart surgery were highly likely to have $\mathrm{CP}$, they were excluded.

Results: Among the 60 patients, 11 (18\%) exhibited a plateau pattern of the right ventricular waveform and had a mean EAT value of 4.2 $\mathrm{mm}$, which was significantly higher than that of patients without such a pattern $(2.1 \mathrm{~mm}, \mathrm{P}<0.001)$. Similarly, the mean body mass index (BMI) values were significantly higher in patients with a plateau pattern than in those without it $\left(27.2 \mathrm{vs} .21 .8 \mathrm{~kg} / \mathrm{m}^{2}, \mathrm{P}<0.001\right)$. EAT was significantly correlated with the BMI $(r=0.72, \mathrm{P}<0.001)$. In patients with a plateau pattern, the triglyceride (TG) and low-density lipoprotein cholesterol (LDL-C) levels were significantly higher (TG: 150 vs. $100 \mathrm{mg} / \mathrm{dL}, \mathrm{LDL}-\mathrm{C}: 121 \mathrm{vs} .95 \mathrm{mg} / \mathrm{dL}, \mathrm{P}<0.05)$ and the left atrial diameter (52.8 vs. $44.7 \mathrm{~mm}, \mathrm{P}<0.01)$ and left atrial volume index $\left(58.7\right.$ vs. $\left.47.6 \mathrm{~mL} / \mathrm{m}^{2}, \mathrm{P}<0.05\right)$ were significantly greater than those in the patients without it. The EAT was also significantly correlated with the TG level $(r=0.37, \mathrm{P}<0.01)$.

Conclusions: Obese patients may present with constrictive hemodynamics, suggesting left ventricular diastolic dysfunction. EAT was significantly correlated with metabolic syndrome components, name-

Manuscript submitted April 7, 2019, accepted April 29, 2019

aDepartment of Cardiology, Japan Community Healthcare Organization Osaka Hospital, 4-2-78, Fukushima, Fukushima-ku, Osaka 553-0003, Japan

${ }^{b}$ Corresponding Author: Yoh Arita, Department of Cardiology, Japan Community Healthcare Organization Osaka Hospital, 4-2-78, Fukushima, Fukushimaku, Osaka 553-0003, Japan. Email: arita-yo@osaka.jcho.go.jp

doi: https://doi.org/10.14740/cr865 ly obesity and TG levels.

Keywords: Right ventricular waveform; Right heart catheterization; Plateau pattern; Obesity; Metabolic syndrome; Diastolic dysfunction

\section{Introduction}

In modern medicine, right heart catheterization (RHC) continues to serve as a useful tool to measure cardiac events in normal and disease states owing to its accuracy and convenience [1]. This technique is currently used to measure the pressure and cardiac outputs, directly inject drugs, and facilitate angiographic visualization with contrast media. Ventricular waveforms are characterized by a "square root sign" or dipand-plateau pattern during diastole due to an abrupt termination of ventricular filling because of pericardial constriction in patients with conditions such as constrictive pericarditis (CP). However, constrictive hemodynamics is not specifically caused by CP. In fact, it is believed to be associated with restrictive cardiomyopathy, right ventricular (RV) infarctions, and acute heart failure [2-4]. However, few studies have reported the relationship between obesity and the RHC waveform. Therefore, this study investigated 60 consecutive Japanese patients who underwent RHC to analyze the correlation between obesity and constrictive hemodynamics presenting with a plateau pattern of the RV waveform.

\section{Methods}

\section{Study population}

A total of 72 consecutive Japanese patients (mean age, 69.5 years; $45 \%$ women) who underwent RHC at the Japan Community Healthcare Organization Osaka Hospital (Osaka, Japan) from July 2016 to September 2018 were included in this study. Patients who had constrictive cardiomyopathy, severe tricuspid regurgitation, and acute heart failure were excluded. In addition, those who underwent open-heart surgery were excluded owing to the high possibility of the presence of CP. Finally, 60 patients were analyzed in this study. Their clinical diagnoses on examination included valvular heart disease in 13 patients $(22 \%)$, ischemic heart disease in nine $(15 \%)$, arrhyth- 
mias in nine (15\%), dilated cardiomyopathy in seven (12\%), hypertensive heart disease in five $(8 \%)$, congenital heart disease in four $(7 \%)$, pulmonary hypertension in three $(5 \%)$, and others in $10(16 \%)$. Regarding the heart failure classification on examination, heart failure with a preserved ejection fraction (HFpEF) was observed in 29 patients $(48 \%)$, heart failure with mid-range ejection fraction (HFmrEF) in eight (13\%), and heart failure with a reduced ejection fraction (HFrEF) in 23 (38\%). This study was conducted in compliance with the ethical standards of the responsible institution on human subjects as well as with the Helsinki Declaration.

\section{Hemodynamic cardiac catheterization}

Standardized right cardiac catheterization was performed in all patients in the fasting state. A Swan-Ganz catheter (Ref 096F6, Edwards Lifesciences Corp., CA, USA) was used to obtain the right-sided cardiac pressure waveforms. The pressures were recorded during normal quiet respirations to measure the endexpiratory pressures. Dip-and-plateau pattern often defined the end-diastolic pressure more than one-third of systolic pressure in many cases. However, there was no report that defined plateau pattern of the ventricular waveform in detail to our knowledge. Therefore, in this paper, we defined the plateau patterns of the diastolic pressure immediately after dip and the enddiastolic pressure just before the onset of the QRS wave are less than $1 \mathrm{~mm} \mathrm{Hg}$.

\section{Measurement of epicardial fat and Doppler echocardiog- raphy}

The epicardial fat thickness was measured by two-dimensional echocardiography in the standard parasternal long-axis view using a commercially available echocardiography machine (Vivid 7, General Electric Healthcare, WI, USA, or iE 33, Phillips Medical Systems, NV, USA) [5]. Epicardial fat was identified as an echo-free space between the outer wall of the myocardium and visceral layer of the pericardium (Fig. 1). Its thickness was measured on the RV free wall during enddiastole. The left ventricular (LV) mass was calculated using a validated formula and indexed for the body surface area (BSA) and height [6]. The LV relative wall thickness was calculated using this formula: (posterior wall thickness $\times 2$ )/end-diastolic diameter. The LV ejection fraction was calculated using the Teichholz formula for patients without LV asynergy and biplane modified Simpson's rule for those with LV asynergy. The mitral flow velocity, peak early diastolic velocity (E), peak late diastolic velocity (A), and E/A ratio were measured using pulsed-wave Doppler. The left atrial volume was measured using the modified biplane area-length method, and the left atrial volume index (LAVI) was corrected for the BSA [7].

\section{Statistical analysis}

The statistical analyses were performed using EZR (Saitama

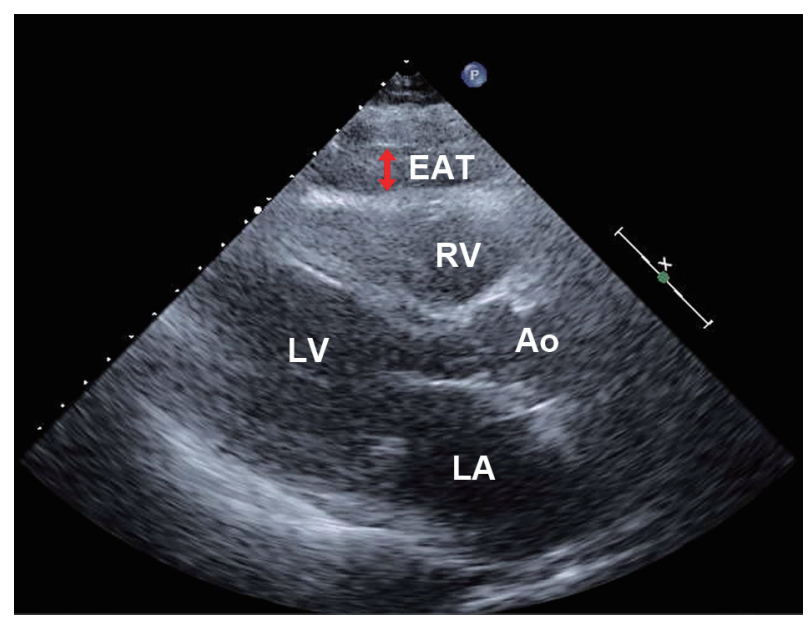

Figure 1. Measurement of the EAT using echocardiography. EAT was detected as an echo-free space between the outer wall of the right ventricle and visceral layer of the pericardium during the end-diastolic cardiac cycle (double arrow). Ao: aorta; EAT: epicardial adipose tissue; LA: left atrium; LV: left ventricle; RV: right ventricle.

Medical Center, Jichi Medical University, Saitama, Japan) [8]. Two-sided $\mathrm{P}$ values of $<0.05$ were considered to indicate statistical significance. Data are expressed as the mean \pm SD or number (\%). Continuous variables were compared using a Student's $t$-test, whereas proportions were compared using a Fisher's extract test.

\section{Results}

The patient characteristics are summarized in Table 1. Among the 60 patients, $11(18 \%)$ exhibited a plateau pattern in their RV waveforms (Fig. 2). Notably, no difference was noted in terms of the sex, age, or height. The mean epicardial adipose tissue (EAT) value in the patients with a plateau pattern was $4.26 \pm 3.13 \mathrm{~mm}$, which was significantly higher than that in the patients without this pattern $(2.19 \pm 1.97 \mathrm{~mm}, \mathrm{P}<0.001$; Fig. $3 \mathrm{a})$. The mean body mass index (BMI) values in the patients with a plateau pattern were significantly higher than that in those without it $\left(27.2 \pm 5.7\right.$ vs. $21.8 \pm 3.6 \mathrm{~kg} / \mathrm{m}^{2}, \mathrm{P}<0.001$; Fig. 3b). Further, the EAT was found to be significantly correlated with the BMI $(r=0.72, \mathrm{P}<0.001$; Fig. 3c). The blood pressures and heart rates did not differ between the two groups. When comparing the lipid profiles, the triglyceride (TG) and low-density lipoprotein cholesterol (LDL-C) levels were found to be significantly higher in patients with a plateau pattern than in those without it (TG: $150.4 \pm 82.6$ vs. $100.2 \pm 67.9$ $\mathrm{mg} / \mathrm{dL}$, LDL-C: $121.8 \pm 35.6$ vs. $95.8 \pm 34.1 \mathrm{mg} / \mathrm{dL}, \mathrm{P}<0.05$; Fig. 4a, b). A significant correlation was observed between the EAT and TG level $(r=0.37, \mathrm{P}<0.01$; Fig. $4 \mathrm{c})$. Moreover, the TG level was significantly correlated with the BMI $(r=0.40$, $\mathrm{P}<0.01$; Fig. 4d). Further, the glycated hemoglobin (HbAlc) and postprandial plasma glucose levels did not differ between the two groups.

The patient catheterization characteristics are summarized in Table 2. The difference in terms of the right cardiac cath- 
Table 1. Comparison of the Clinical and Laboratory Characteristics of Patients Without and With a Plateau Pattern

\begin{tabular}{llll}
\hline & Without a plateau $(\mathbf{n}=\mathbf{4 9})$ & With a plateau $(\mathbf{n}=\mathbf{1 1})$ & P value \\
\hline Age $($ years $)$ & $69.3 \pm 13.4$ & $69.9 \pm 16.8$ & 0.90 \\
Male & $28(57 \%)$ & $5(45 \%)$ & 0.52 \\
Weight $(\mathrm{kg})$ & $55.5 \pm 12.2$ & $69.0 \pm 14.2$ & $<0.01$ \\
Height $(\mathrm{m})$ & $1.58 \pm 0.09$ & $1.59 \pm 0.08$ & $<.89$ \\
BMI $\left(\mathrm{kg} / \mathrm{m}^{2}\right)$ & $21.8 \pm 3.6$ & $27.2 \pm 5.7$ & $<0.001$ \\
EAT $(\mathrm{mm})$ & $2.19 \pm 1.97$ & $4.26 \pm 3.13$ & 0.61 \\
Systolic BP $(\mathrm{mm} \mathrm{Hg})$ & $129.2 \pm 23.5$ & $133.2 \pm 23.0$ & 0.06 \\
Diastolic BP $(\mathrm{mm} \mathrm{Hg})$ & $67.7 \pm 13.6$ & $76.7 \pm 16.0$ & 0.56 \\
Heart rate (beats/min) & $70.8 \pm 14.3$ & $73.6 \pm 15.1$ & 0.08 \\
TC $(\mathrm{mg} / \mathrm{dL})$ & $170.3 \pm 42.2$ & $196.2 \pm 47.9$ & 0.34 \\
HDL-C (mg/dL) & $57.7 \pm 18.5$ & $51.1 \pm 27.3$ & $<0.05$ \\
LDL-C (mg/dL) & $95.8 \pm 34.1$ & $121.8 \pm 35.6$ & $<0.05$ \\
TG (mg/dL) & $100.2 \pm 67.9$ & $150.4 \pm 82.6$ & 0.75 \\
HbAlc (\%) & $6.4 \pm 1.59$ & $6.2 \pm 0.77$ & 0.79 \\
PPG (mg/dL) & $126 \pm 62.8$ & $120 \pm 27.9$ & \\
\hline
\end{tabular}

Data are expressed as the mean \pm SD or $n(\%)$. BMI: body mass index; BP: blood pressure; EAT: epicardial adipose tissue; HbA1c: glycated hemoglobin; HDL-C: high-density lipoprotein cholesterol; LDL-C: low-density lipoprotein cholesterol; PPG: postprandial plasma glucose; TC: total cholesterol; TG: triglyceride.

eterization parameters between the patients with and without a plateau pattern in their RV waveforms was not significant.

Regarding the echocardiographic characteristics (Table 3), the left atrial diameter was significantly larger in the patients with a plateau pattern than in those without it $(52.8 \pm 4.30 \mathrm{~mm}$ vs. $44.7 \pm 8.69 \mathrm{~mm}, \mathrm{P}<0.01$; Fig. 5a). Similarly, the LAVI was significantly higher in the patients with a plateau pattern than in those without it $\left(58.7 \pm 12.1 \mathrm{~mL} / \mathrm{m}^{2}\right.$ vs. $47.6 \pm 14.9 \mathrm{~mL} / \mathrm{m}^{2}$, $\mathrm{P}<0.05$; Fig. 5b).

\section{Discussion}

The normal RV systolic and end-diastolic pressures are 20 - 30 and $0-8 \mathrm{~mm} \mathrm{Hg}$, respectively. RV pressure tracings are characterized by ventricular waveforms with a rapid pressure increase during ventricular contractions and rapid pressure decay

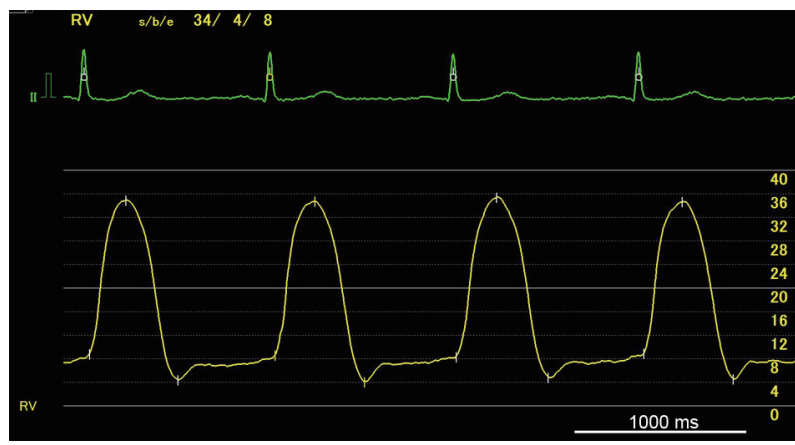

Figure 2. A representative plateau pattern of the right ventricular waveform. during relaxation, with the diastolic phase characterized by an initially low but gradually increasing pressure [9]. On the other hand, ventricular waveforms are characterized by a "square root sign" or dip-and-plateau pattern in patients with conditions such as CP. In addition to $\mathrm{CP}$, constrictive hemodynamics is caused by restrictive cardiomyopathy, RV infarctions, and acute heart failure [2-4]. Obesity is also considered a cause of constrictive hemodynamics $[10,11]$. However, studies reporting the relationship between obesity and RHC waveforms are limited. Therefore, this study demonstrated that patients with obesity were more likely to exhibit a plateau pattern in the RV waveform than those without obesity in Japan. To the best of our knowledge, this is the first study to report this result.

EAT is the visceral adipose tissue depot mainly located around the epicardial coronary vessels and is present in the heart and interspersed with the myocardial muscle fibers [12]. EAT may mediate the deleterious effects of obesity and myocardial inflammation $[5,13,14]$. The most common myocardial disease in obese individuals is HFpEF [15]. Several studies have reported a significant relationship between EAT and the BMI [12]. Different cut-off points of the epicardial fat thickness have been proposed for predicting coronary artery disease, metabolic syndrome, insulin resistance, excess visceral fat accumulation, and subclinical atherosclerosis [5]. In our study, the EAT and BMI were found to be significantly correlated, confirming the results of previous reports. The lipid profiles comprised of the TG and LDL-C levels were also significantly higher in the patients with a plateau pattern than in those without it. TGs, one of the metabolic syndrome components, were found to be correlated with the EAT, suggesting that patients with obesity exhibiting a plateau pattern in the RV waveforms have high TG levels and possibly suffer from 
a

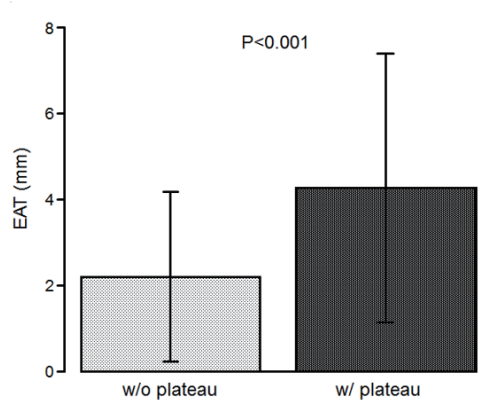

b

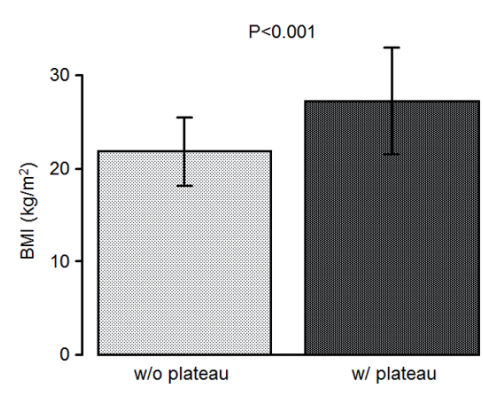

C

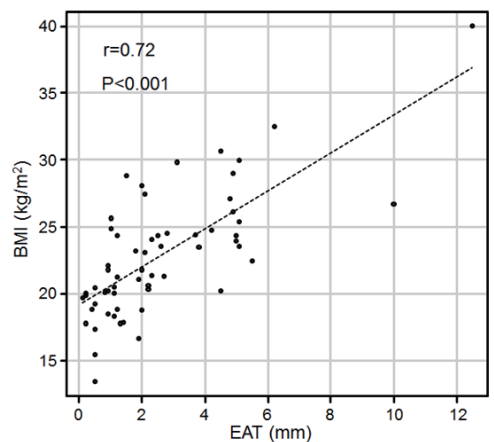

Figure 3. (a) The EAT values in the patients who exhibited a plateau pattern (w/plateau) are significantly higher than those in the patients who did not (w/o plateau). (b) The mean BMl values are higher in the patients with a plateau than in those without it. (c) Scatter plots between the EAT and BMI. BMI: body mass index; EAT: epicardial adipose tissue.

metabolic syndrome.

Obesity is also reported as one of the major risk factors for LV diastolic dysfunction [16-20]. A marked increase in the epicardial fat thickness may impair the myocardial relaxation and alter the ventricular diastolic filling [21]. The LAVI is thought to be one of the parameters of LV diastolic dysfunction [22]. In our study, the LAVI was significantly higher in the patients with

a

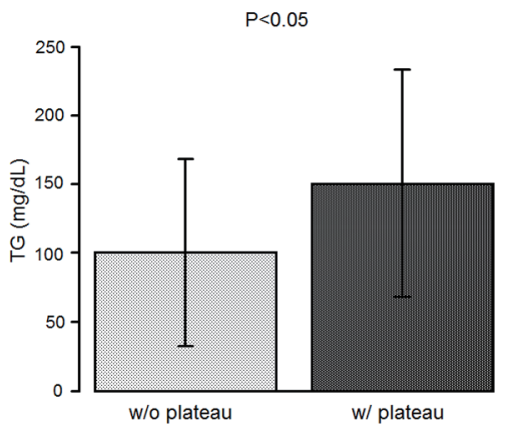

C

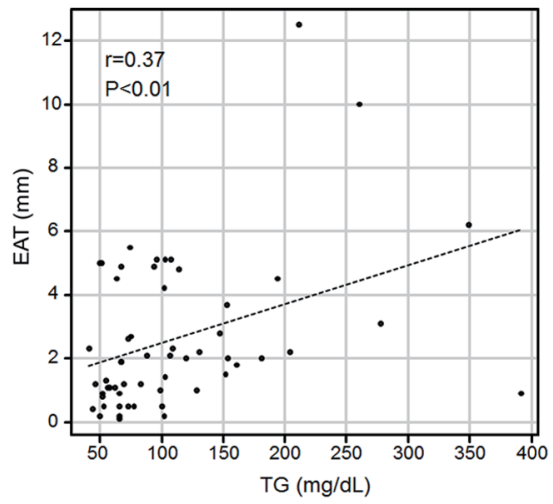

a plateau pattern than in those without it, indicating that obesity is associated with LV diastolic dysfunction. Epicardial fat is considered to physically limit the heart expansion. In previous studies, EAT has been shown to be significantly correlated with LV diastolic function, and is expressed as E/A, mitral annulus early peak velocity (e'), and E/e' [16, 23]. However, our data differed from that of the previous reports. A possible reason for

\section{b}

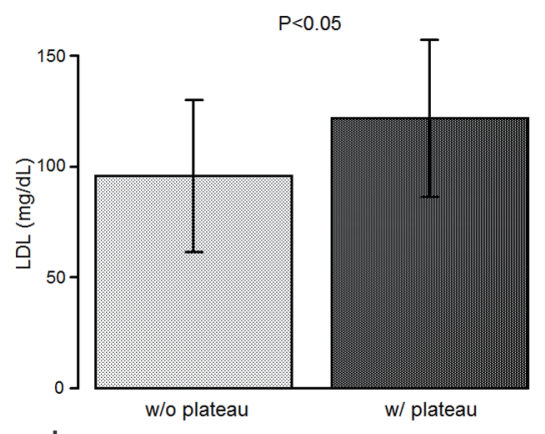

d

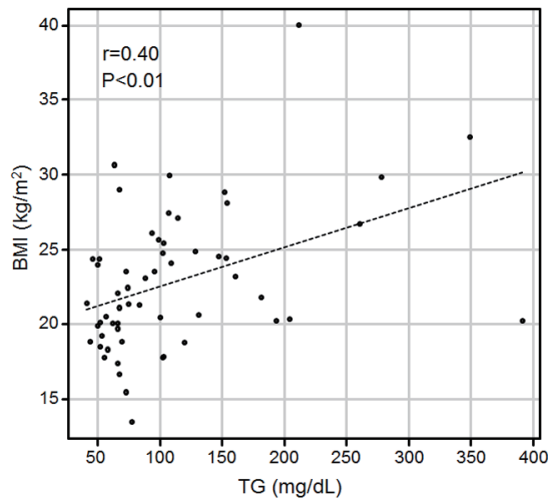

Figure 4. (a) The TG levels in the patients who exhibited a plateau pattern (w/plateau) are significantly higher than in those who did not (w/o plateau). (b) The LDL-C levels in the patients with a plateau are significantly higher than those in the patients without a plateau. (c) Scatter plots between the TG level and EAT. (d) Scatter plots between the TG level and BMI. BMI: body mass index; EAT: epicardial adipose tissue; LDL-C: low-density lipoprotein cholesterol; TG: triglyceride. 
Table 2. Comparison of the Right Cardiac Catheterization Characteristics of Patients Without and With a Plateau Pattern

\begin{tabular}{llll} 
& Without a plateau $(\mathbf{n}=49)$ & With a plateau $(\mathbf{n}=11)$ & P value \\
\hline Cardiac output $(\mathrm{L} / \mathrm{min})$ & $4.93 \pm 1.37$ & $5.18 \pm 1.73$ & 0.61 \\
Cardiac index $\left(\mathrm{L} / \mathrm{min} / \mathrm{m}^{2}\right)$ & $3.21 \pm 0.94$ & $2.97 \pm 0.82$ & 0.46 \\
Main PA systolic pressure (mm Hg) & $33.5 \pm 12.8$ & $35.7 \pm 10.1$ & 0.63 \\
Main PA diastolic pressure (mm Hg) & $15.9 \pm 7.19$ & $16.3 \pm 6.5$ & 0.88 \\
Main PA mean pressure (mm Hg) & $23.0 \pm 9.51$ & $23.8 \pm 9.11$ & 0.81 \\
Right PA systolic pressure (mm Hg) & $33.3 \pm 12.4$ & $35.7 \pm 10.0$ & 0.56 \\
Right PA diastolic pressure (mm Hg) & $16.4 \pm 7.11$ & $15.7 \pm 6.88$ & 0.75 \\
Right PA mean pressure (mm Hg) & $22.9 \pm 9.39$ & $23.7 \pm 9.18$ & 0.79 \\
Mean PCWP (mm Hg) & $14.4 \pm 7.61$ & $14.3 \pm 6.63$ & 0.96 \\
RV systolic pressure (mm Hg) & $33.0 \pm 11.7$ & $33.2 \pm 10.9$ & 0.94 \\
RV diastolic pressure (mm Hg) & $5.67 \pm 4.39$ & $4.54 \pm 2.20$ & 0.41 \\
RV end-diastolic pressure (mm Hg) & $9.00 \pm 4.07$ & $9.18 \pm 3.68$ & 0.89 \\
Mean RA (mm Hg) & $6.59 \pm 3.85$ & $6.81 \pm 3.06$ & 0.85
\end{tabular}

Data are expressed as the mean \pm SD. PA: pulmonary artery; PCWP: pulmonary capillary wedge pressure; RA: right atrium; RV: right ventricle.

this discrepancy was that our study included patients with a reduced EF. Because HFpEF and HFrEF represent two different obstacles in the heart failure spectrum, they may influence various markers representing extended disturbances [24].

This study showed that some patients have a plateau pattern in the RV waveform even in the absence of diseases that causes constrictive hemodynamics, which indicated that it occurs due to obesity in some patients.
EAT measurements using echocardiography have several advantages such as their easy availability and low cost; however, some limitations are inevitable because echocardiography is not necessarily the optimal tool to quantify the epicardial fat thickness. Echocardiographic measurement techniques also vary widely, which results in inconsistencies among studies because the epicardial fat distribution is focally asymmetric. Additionally, several heart diseases such as restrictive cardio-

Table 3. Comparison of the Echocardiographic Characteristics of Patients Without and With a Plateau Pattern

\begin{tabular}{llll}
\hline & Without a plateau $(\mathbf{n}=\mathbf{4 9})$ & With a plateau $(\mathbf{n}=\mathbf{1 1})$ & P value \\
\hline LVDd $(\mathrm{mm})$ & $51.2 \pm 9.87$ & $48.5 \pm 8.53$ & 0.40 \\
LVDs $(\mathrm{mm})$ & $37.8 \pm 12.2$ & $35.0 \pm 10.6$ & 0.48 \\
IVSd $(\mathrm{mm})$ & $10.9 \pm 2.16$ & $11.4 \pm 1.17$ & 0.42 \\
PWd $(\mathrm{mm})$ & $10.4 \pm 1.92$ & $11.1 \pm 0.50$ & 0.27 \\
EF $(\%)$ & $49.4 \pm 20.5$ & $50.1 \pm 19.5$ & 0.91 \\
LVMI $\left(\mathrm{g} / \mathrm{m}^{2}\right)$ & $141.4 \pm 70.6$ & $115.7 \pm 32.9$ & 0.26 \\
RWT & $0.435 \pm 0.096$ & $0.476 \pm 0.069$ & 0.21 \\
E/A ratio & $1.28 \pm 0.69$ & $1.60 \pm 0.92$ & 0.40 \\
DT $(\mathrm{ms})$ & $187.5 \pm 60.4$ & $173.0 \pm 48.0$ & 0.52 \\
e' $(\mathrm{cm} / \mathrm{s})$ & $5.66 \pm 2.16$ & $7.15 \pm 1.81$ & 0.11 \\
E/e' $(\mathrm{ratio})$ & $16.3 \pm 7.95$ & $16.5 \pm 6.14$ & 0.95 \\
LAD $(\mathrm{mm})$ & $44.7 \pm 8.69$ & $52.8 \pm 4.30$ & $<0.01$ \\
LAVI $\left(\mathrm{mL} / \mathrm{m}^{2}\right)$ & $47.6 \pm 14.9$ & $58.7 \pm 12.1$ & $<0.05$ \\
IVC ex $(\mathrm{mm})$ & $15.8 \pm 4.68$ & $17.8 \pm 6.75$ & 0.25 \\
IVC in $(\mathrm{mm})$ & $6.87 \pm 4.99$ & $10.0 \pm 8.49$ & 0.09 \\
IVCCI & $58.9 \pm 20.1$ & $50.2 \pm 24.9$ & 0.25 \\
\hline
\end{tabular}

Data are expressed as the mean \pm SD. DT: mitral deceleration time; e': mitral annulus early peak velocity; EF: ejection fraction; IVC ex: inferior vena cava expiration; IVC in: inferior vena cava inspiration; IVCCI: IVC collapsibility index; IVSd: interventricular septum diameter; LAD: left atrial diameter; LAVI: left atrial volume index; LVDd: left ventricular end-diastolic diameter; LVDs: left ventricular end-systolic diameter; LVMI: left ventricular mass index; PWd: posterior wall diameter; RWT: relative wall thickness. 


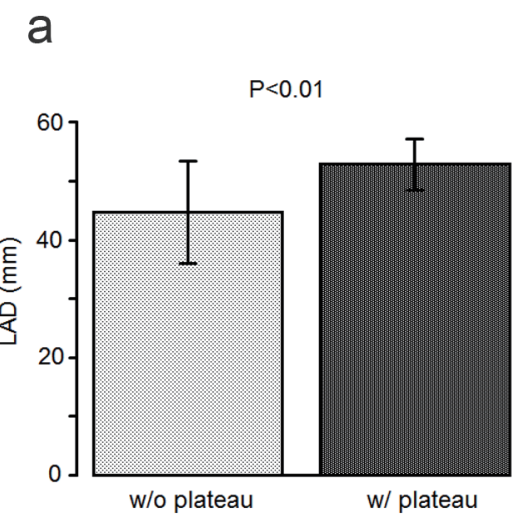

\section{b}

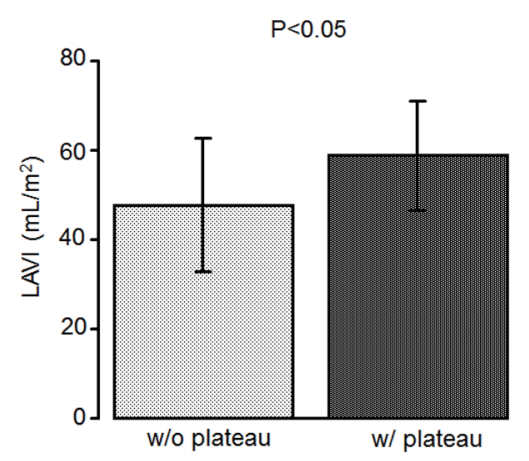

Figure 5. (a) The left atrial diameter is significantly larger in the patients with a plateau pattern (w/plateau) than in those without it (w/o plateau). (b) The left atrial volume index is significantly higher in the patients with a plateau pattern than in those without it.

myopathy were not completely excluded in this study because an endomyocardial biopsy was not performed in all patients.

\section{Conclusions}

Patients with obesity may present with constrictive hemodynamics due to LV diastolic dysfunction. The EAT was significantly correlated with the metabolic syndrome components such as obesity and the TG level.

\section{Acknowledgments}

None.

\section{Financial Disclosure}

None.

\section{Conflict of Interest}

The authors declare that there is no conflict of interest.

\section{Informed Consent}

All patients provided written informed consent.

\section{Author Contributions}

All authors contributed to data acquisition and analysis.

\section{References}

1. Nossaman BD, Scruggs BA, Nossaman VE, Murthy SN,
Kadowitz PJ. History of right heart catheterization: 100 years of experimentation and methodology development. Cardiol Rev. 2010;18(2):94-101.

2. Meaney E, Shabetai R, Bhargava V, Shearer M, Weidner C, Mangiardi LM, Smalling R, et al. Cardiac amyloidosis, contrictive pericarditis and restrictive cardiomyopathy. Am J Cardiol. 1976;38(5):547-556.

3. Raabe DS, Jr., Chester AC. Right ventricular infarction. Chest. 1978;73(1):96-99.

4. Lavine SJ, Campbell CA, Kloner RA, Gunther SJ. Diastolic filling in acute left ventricular dysfunction: role of the pericardium. J Am Coll Cardiol. 1988;12(5):13261333.

5. Iacobellis G, Willens HJ. Echocardiographic epicardial fat: a review of research and clinical applications. J Am Soc Echocardiogr. 2009;22(12):1311-1319; quiz 1417-1318.

6. Devereux RB, Casale PN, Kligfield P, Eisenberg RR, Miller D, Campo E, Alonso DR. Performance of primary and derived M-mode echocardiographic measurements for detection of left ventricular hypertrophy in necropsied subjects and in patients with systemic hypertension, mitral regurgitation and dilated cardiomyopathy. Am J Cardiol. 1986;57(15):1388-1393.

7. Patel DA, Lavie CJ, Milani RV, Ventura HO. Left atrial volume index predictive of mortality independent of left ventricular geometry in a large clinical cohort with preserved ejection fraction. Mayo Clin Proc. 2011;86(8):730737.

8. Kanda Y. Investigation of the freely available easy-touse software 'EZR' for medical statistics. Bone Marrow Transplant. 2013;48(3):452-458.

9. Hansen AT, Eskildsen P, Gotzsche H. Pressure curves from the right auricle and the right ventricle in chronic constrictive pericarditis. Circulation. 1951;3(6):881-888.

10. Do GW, Ku BS, Park CS, Kim SJ, Shin ES, Choi SH, Lee SG. A case of constrictive pericarditis associated with huge epicardial fat volume. Korean Circ J. 2009;39(3):116-120.

11. Pilz B, Brasen JH, Schneider W, Luft FC. Obesity and hypertension-induced restrictive cardiomyopathy: a harbinger of things to come. Hypertension. 2004;43(5):911- 
917.

12. Rabkin SW. The relationship between epicardial fat and indices of obesity and the metabolic syndrome: a systematic review and meta-analysis. Metab Syndr Relat Disord. 2014;12(1):31-42.

13. Packer M. Epicardial Adipose Tissue May Mediate Deleterious Effects of Obesity and Inflammation on the Myocardium. J Am Coll Cardiol. 2018;71(20):2360-2372.

14. Jeong JW, Jeong MH, Yun KH, Oh SK, Park EM, Kim YK, Rhee SJ, et al. Echocardiographic epicardial fat thickness and coronary artery disease. Circ J. 2007;71(4):536-539.

15. Kitzman DW, Shah SJ. The HFpEF Obesity Phenotype: The Elephant in the Room. J Am Coll Cardiol. 2016;68(2):200-203.

16. Nakanishi K, Fukuda S, Tanaka A, Otsuka K, Taguchi $\mathrm{H}$, Shimada K. Relationships between periventricular epicardial adipose tissue accumulation, coronary microcirculation, and left ventricular diastolic dysfunction. Can J Cardiol. 2017;33(11):1489-1497.

17. Wong CY, O'Moore-Sullivan T, Leano R, Byrne N, Beller E, Marwick TH. Alterations of left ventricular myocardial characteristics associated with obesity. Circulation. 2004;110(19):3081-3087.

18. Russo C, Jin Z, Homma S, Rundek T, Elkind MS, Sacco RL, Di Tullio MR. Effect of obesity and overweight on left ventricular diastolic function: a community- based study in an elderly cohort. J Am Coll Cardiol. 2011;57(12):1368-1374.

19. Share BL, La Gerche A, Naughton GA, Obert P, Kemp JG. Young women with abdominal obesity have subclinical myocardial dysfunction. Can J Cardiol. 2015;31(9):11951201.

20. Pascual M, Pascual DA, Soria F, Vicente T, Hernandez AM, Tebar FJ, Valdes M. Effects of isolated obesity on systolic and diastolic left ventricular function. Heart. 2003;89(10):1152-1156.

21. Iacobellis G, Leonetti F, Singh N, A MS. Relationship of epicardial adipose tissue with atrial dimensions and diastolic function in morbidly obese subjects. Int J Cardiol. 2007;115(2):272-273.

22. Pritchett AM, Mahoney DW, Jacobsen SJ, Rodeheffer RJ, Karon BL, Redfield MM. Diastolic dysfunction and left atrial volume: a population-based study. J Am Coll Cardiol. 2005;45(1):87-92.

23. Rabkin SW. Is reduction in coronary blood flow the mechanism by which epicardial fat produces left ventricular diastolic dysfunction? Can J Cardiol. 2017;33(11):14591461.

24. Borlaug BA, Redfield MM. Diastolic and systolic heart failure are distinct phenotypes within the heart failure spectrum. Circulation. 2011;123(18):2006-2013; discussion 2014. 